\title{
Dichotomy Theorems for Homomorphism Polynomials of Graph Classes
}

\author{
Christian Engels*
}

November 10, 2018

\begin{abstract}
In this paper, we will show dichotomy theorems for the computation of polynomials corresponding to evaluation of graph homomorphisms in Valiant's model. We are given a fixed graph $H$ and want to find all graphs, from some graph class, homomorphic to this $H$. These graphs will be encoded by a family of polynomials.

We give dichotomies for the polynomials for cycles, cliques, trees, outerplanar graphs, planar graphs and graphs of bounded genus.
\end{abstract}

\section{Introduction}

Graph homomorphisms are studied because they give important generalizations of many natural questions ( $k$-coloring, acyclicity, binary CSP and many more cf. [17]). One of the first results, given by Hell and Nešetřil [16], was on the decision problem where they gave a dichotomy. The exact result was, that deciding if there exists a homomorphism from some graph $G$ to a fixed undirected graph $H$ is polynomial time computable if $H$ is bipartite and NP-complete otherwise. A different side of graph homomorphisms was looked at by Chekuri and Rajaraman [4] Dalmau, Kolaitis and Vardi [6], and Freuder [11] and finally Grohe [14]. They studied the following: Given a restricted graph class $\mathcal{G}$, decide if there is a graph $G \in \mathcal{G}$ homomorphic to a given graph $H$. Instead of restricting the graph $H$ as in the first problem, we restrict the graph classes we map from. Later, focus shifted onto the counting versions of these two sides where we have to count the number of homomorphisms. Dyer and Greenhill [10] solved the first problem in the counting case and Dalmau and Jonsson [5] the second. The first problem was extended by Bulatov and Grohe [1] to graphs with multiple edges. They also notice some interesting connections to statistical physics and constraint satisfaction problems. A good introduction to the history of graph homomorphism was written by Grohe and

${ }^{*}$ Saarland University, Department of Computer Science

engels@cs.uni-saarland.de 
Thurley [15] and research on these topics continues even today with two noticeable being the works by Goldberg, Grohe, Jerrum and Thurley [12] and by Cai, Chen and Lu [3].

However, the arithmetic circuit complexity was still open. The previous results could only show that the hard cases have no polynomial size circuits for counting the number of homomorphisms but it was unclear if these problems are VNP complete. The study of VNP complete problems and the arithmetic world was started in the seminal paper by Valiant [22]. In this world, we look at the complexity of computing a family of polynomials using a family of arithmetic circuits. Recently, a dichotomy for graph homomorphisms was shown by Rugy-Altherre [7. Here a graph is encoded by a product of edge variables and sets of graphs as sums over these products. This is known as generating function and a detailed definition will be provided in Section 2. However, this result was for the first side of the graph homomorphism problem.

In this paper we look at the second side of the graph homomorphism problem to complete the picture for the arithmetic circuit world. While we could not get a general theorem as in [5], we show multiple hardness proofs for some classes. We will look at cycles, cliques, trees, outerplanar graphs, planar graphs and graphs of bounded genus.

Recently, homomorphism polynomials in a different form are even used for giving natural characterizations of VP independent of the circuit definition [9]. In this way our results can be interpreted as showing that some straightforward candidates originating from the counting world do not give a characterization of VP.

Section 2 gives a formal introduction to our model, related hard problems and states the problem precisely. We prove our dichotomies in Sections 3.1 to 3.6 where the constructions in Sections 3.4 to 3.6 build on each other. The construction in Section 3.3 will use a slightly different model as the other sections. We will give a brief introduction into concepts from graph genus in Section 3.6 but refer the reader to the textbook by Diestel [8].

\section{Model and Definitions}

Let us first give a brief introduction to the field of Valiant's classes. For further information the reader is referred to the textbook by Bürgisser [2]. In this theory, we are given an arithmetic circuit (a directed acyclic connected graph) with addition and multiplication gates over some field $K$. These gates are either connected to other gates or input gates from the set $K \cup X$ for some set of indeterminates $X$. At the end we have exactly one output gate. An arithmetic circuit computes a polynomial in $K[X]$ at the output gate in the obvious way.

As Valiant's model is non-uniform, a problem consists of families of polynomials. A $p$-family is a sequence of polynomials $\left(f_{n}\right)$ over $K[X]$ where the number of variables is $n$ and the degree is bounded by some polynomial in $n$. Additionally the family of polynomials $\left(f_{n}\right)$ should be computed by a family of arithmetic circuits $\left(C_{n}\right)$ where $f_{n}$ is computed by $C_{n}$ for all $n$. Valiant's Model focuses its study on $p$-families of polynomials.

We define $L(f)$ to be the number of gates for a minimal arithmetic circuit computing a given polynomial $f \in K[X]$. VP is the class of all $p$-families of polynomi- 
als where $L\left(f_{n}\right)$ is bounded polynomially in $n$. Let $q(n), r(n), s(n)$ be polynomially bounded functions. A $p$-family $\left(f_{n}\right) \in K\left[x_{1}, \ldots, x_{q(n)}\right]$ is in VNP if there exists a family $\left(g_{n}\right) \in K\left[x_{1}, \ldots, x_{r(n)}, y_{1}, \ldots, y_{s(n)}\right]$ in VP such that

$$
f\left(x_{1}, \ldots, x_{q(n)}\right)=\sum_{\epsilon \in\{0,1\}^{s(n)}} g\left(x_{1}, \ldots, x_{r(n)}, \epsilon_{1}, \ldots, \epsilon_{s(n)}\right) .
$$

The classes VP and VNP are considered algebraic analogues to $\mathrm{P}$ and $\mathrm{NP}$ or more accurately $\# \mathrm{P}$. We can also define an algebraic version of $\mathrm{AC}_{0}$, mentioned by Mahajan and Rao [18. A $p$-family is in $\mathrm{VAC}_{0}$ if there exists a family of arithmetic circuit of constant depth and polynomial size with unbounded fan-in that computes the family of polynomials.

The notion of a reduction in Valiant's model is given by $p$-projections. A $p$-family $\left(f_{n}\right)$ is a $p$-projection of $\left(g_{n}\right)$, written as $\left(f_{n}\right) \leq_{p}\left(g_{n}\right)$, if there exists a polynomially bounded function $q(n)$ such that for every $n, f\left(x_{1}, \ldots, x_{n}\right)=g\left(a_{1}, \ldots, a_{q(n)}\right)$ for some $a_{i} \in K \cup\left\{x_{1}, \ldots, x_{n}\right\}$. Once we have a reduction, we get a notion of completeness in the usual way.

However, we use a different kind of reduction called a $c$-reduction. This is similar to a Turing reduction in the Boolean world. We define $L^{g}(f)$ as the number of gates for computing $f$ where the arithmetic circuits is enhanced with an oracle gate for $g$. An oracle gate for the polynomial $g \in K\left[x_{1}, \ldots, x_{n^{\prime}}\right]$ has as output $g\left(a_{1}, \ldots, a_{n^{\prime}}\right)$ where $a_{1}, \ldots, a_{n^{\prime}}$ are the inputs to this gate. This allows us to evaluate $g$ on $a_{1}, \ldots, a_{n^{\prime}}$ in one step if we computed $a_{1}, \ldots, a_{n^{\prime}}$ previously in our circuit.

We say $f c$-reduces to $g$, written $\left(f_{n}\right) \leq_{c}\left(g_{n}\right)$, if there exists a polynomial $p$ such that $L^{g_{p(n)}}(f)$ is bounded by some polynomial. This reduction, however, is only useful for $\mathrm{VNP}$ and not for $\mathrm{VAC}_{0}$ and VP. In this paper we will exclusively deal with $c$-reductions for our VNP completeness results.

\subsection{Complete Problems}

We continue with the basic framework of graph properties. In the following $K$ will be an infinite field.

Definition 2.1. Let $X$ be a set of indeterminates. Let $\mathcal{E}$ be a graph property, that is, a class of graphs which contains with every graph also all of its isomorphic copies. Let $G=(V, E)$ be an edge weighted, undirected graph with a weight function $w: E \rightarrow K \cup X$. We extend the weight function by $w\left(E^{\prime}\right):=\prod_{e \in E^{\prime}} w(e)$ to subsets $E^{\prime} \subseteq E$.

The generating function $\operatorname{GF}(G, \mathcal{E})$ of the property $\mathcal{E}$ is defined as

$$
\operatorname{GF}(G, \mathcal{E}):=\sum_{E^{\prime} \subseteq E} w\left(E^{\prime}\right)
$$

where the sum is over all subsets $E^{\prime}$ such that the subgraph $\left(V, E^{\prime}\right)$ of $G$ has property $\mathcal{E}$.

The reader should notice that the subgraph still contains all vertices and just takes a subset of the edges. 
In the following, let $G$ be a graph and let $X=\left\{x_{e} \mid e \in E\right\}$. We label each edge $e$ by the indeterminate $x_{e}$. We conclude by stating some basic VNP-complete problems. Proofs of these facts can be found in the textbook by Bürgisser [2].

Theorem $2.1([2]) \cdot \operatorname{GF}\left(K_{n}, \mathcal{U H C}_{n}\right)$ is $V N P$-complete where $\mathcal{U H C}_{n}$ is the set of all hamiltonian cycles in $K_{n}$.

Theorem $2.2([2])$. Let $\mathcal{C} \mathcal{L}$ be the set of all cliques. Meaning, the set of all graphs, where one connected component is a complete graph and each of the remaining connected components consist of one vertex only. The family $\mathrm{GF}\left(K_{n}, \mathcal{C L}\right)$ is VNP-complete.

Theorem $2.3([2])$. Let $\mathcal{M}$ be the set of all graphs where all connected components have exactly two vertices. The family $\operatorname{GF}\left(K_{n}, \mathcal{M}\right)$ is VNP-complete.

This polynomial gives us all perfect matchings in a graph. It is well known that the original VNP-complete problem, the permanent, is equal to $\operatorname{GF}\left(K_{n, n}, \mathcal{M}\right)$ for bipartite graphs which is a projection of $\mathrm{GF}\left(K_{n^{2}}, \mathcal{M}\right)$.

\subsection{The problem and related definitions}

We now formulate our problem. Let $G, H$ be undirected graphs. We will generally switch freely between having the variable indexed by either edges $\left(x_{e}\right)$ or vertices $\left(x_{i, j}\right.$ for $i, j \in V)$. We let $x_{j}$ correspond to the self-loop at vertex $j$.

A homomorphism from $G=(V, E)$ to $H=\left(V^{\prime}, E^{\prime}\right)$ is a mapping $f: V \rightarrow V^{\prime}$ such that for all edges $\{u, v\} \in E$ there exist an edge $\{f(u), f(v)\} \in E^{\prime}$. We can define the corresponding generating function as follows.

Definition 2.2. Let $\mathcal{H}_{H}$ be the property of all connected graphs homomorphic to a fixed $H$. We denote by $\mathcal{F}^{H, n}$ the generating function $\mathcal{F}^{H, n}:=\operatorname{GF}\left(K_{n}, \mathcal{H}_{H}\right)$.

We can state now the first dichotomy theorem.

Theorem 2.4 ([7]). If $H$ has a loop or no edges, $\mathcal{F}^{H, n}$ is in $V A C_{0}$ and otherwise it is VNP-complete under c-reductions.

Instead of looking at all graphs, we want to look at a restricted version. What happens if we do not want to find every graph homomorphic to a given $H$ but every cycle homomorphic to a given $H$ ? We state our problem in the next definitions.

Definition 2.3. Let $\mathcal{E}_{n}$ be a graph property. Then $\mathcal{F}_{\mathcal{E}_{n}}^{H, n}$ is the generating function for all graphs in $\mathcal{E}_{n}$ on $n$ vertices homomorphic to a fixed graph $H$.

Definition 2.4. We define the following graph polynomials.

- $\mathcal{F}_{\text {cycle }_{n}}^{H, n}$ where cycle $e_{n}$ is the property where one connected component is a cycle and the others are single vertices in a graph of size $n$.

- $\mathcal{F}_{\text {clique }_{n}}^{H, n}$ where clique ${ }_{n}$ is the property where one connected component is a clique and the others are single vertices in a graph of size $n$. 
- $\mathcal{F}_{\text {tress }_{n}}^{H, n}$ where trees $_{n}$ is the property where one connected component is a tree and the others are single vertices in a graph of size $n$.

- $\mathcal{F}_{\text {outerplanar }_{n}}^{H, n}$ where outerplanar ${ }_{n}$ is the property where one connected component is a outerplanar graph and the others are single vertices in a graph of size $n$.

- $\mathcal{F}_{\text {planar }_{n}}^{H, n}$ where planar $_{n}$ is the property where one connected component is a planar graph and the others are single vertices in a graph of size $n$.

- $\mathcal{F}_{\text {genus }(k), n}^{H, n}$ where genus $(k), n$ is the property where one connected component has genus $k$ and the others are single vertices in a graph of size $n$.

We will use the notation $\mathcal{F}_{\text {cycle }}, \mathcal{F}_{\text {clique }}, \mathcal{F}_{\text {tree }}, \mathcal{F}_{\text {outerplanar }}, \mathcal{F}_{\text {planar }}$ and $\mathcal{F}_{\text {genus }(k)}$ as a shorthand.

Let us now introduce the homogeneous degree of a polynomial.

Definition 2.5. Let $\bar{x}=x_{i_{1}}, \ldots, x_{i_{l}}$ be a subset of variables and $\left(f_{n}\right)$ be a p-family. We can write $f_{n}$ as

$$
f_{n}=\sum_{\bar{i}} \alpha_{\bar{i}} \prod_{j=1}^{n} x_{j}^{i_{j}}
$$

The homogeneous component of $f_{n}$ of degree $k$ with variables $\bar{x}$ is

$$
\operatorname{HOMC}_{k}^{\bar{x}}\left(f_{n}\right)=\sum_{\substack{i_{1}, \ldots, i_{l} \\ k=\sum_{j=1}^{l} i_{j}}} \alpha_{i_{1}, \ldots, l_{l}} x_{i_{1}}^{i_{i}} \ldots x_{i_{l}}^{i_{j}} .
$$

Finally, we need a last lemma in our proofs. This lemma was stated explicit by RugyAltherre [7] and can also be found in [2]. It will give us a way to extract all polynomials of homogeneous degree $k$ in some set of variables in $c$-reductions.

Lemma 2.1. Then for any sequence of integers $\left(k_{n}\right)$ there exists a c-reduction from the homogeneous component to the polynomial itself:

$$
\operatorname{HOMC}_{k_{n}}^{\bar{x}}\left(f_{n}\right) \leq_{c}\left(f_{n}\right) .
$$

The circuit for the reduction has size in $\mathcal{O}\left(n \delta_{n}\right)$ where $\delta_{n}$ is the degree of $f_{n}$.

The reader should note that using this theorem will blow up our circuit polynomially in size and can hence be used only a constant number of times in succession. However, we can use this lemma on subsets of vertices. We replace every variable $x_{i}$ in the subset by $x_{i} y$ for a new variable $y$ and take the homogeneous components of $y$. We will use this technique to enforce edges to be taken. Notice that enforcing $n$ edges to be taken only increases the circuit size by a factor of $n$. Additionally, we can set edge variables to zero to deny our polynomial using these edges.

Let $G$ be a graph that is homomorphic to a given $H$. We will, in general, ignore self-loops in $G$, i.e. assume $G$ to never have any self-loops. If we have proven a theorem for all $G$ without self loops, we can just take the homomorphism polynomial with selfloops, take the homogeneous component of degree zero of all self-loops and get the homomorphism polynomial without self-loops. As we will prove the dichotomy for these, the hardness will follow. 


\section{Dichotomies}

\subsection{Cycles}

As a first graph class we look at cycles. The proof for the dichotomy will be relatively easy and gives us a nice example to get familiar with homomorphism polynomials and hardness proofs. Our proofs will, in general, reason first about the kind of monomials that exist for a given $H$ and then try to extract or modify these via Lemma 2.1 to get a solution to a VNP-complete problem. This will yield the reduction.

Our main dichotomy for cycles is the following theorem.

Theorem 3.1. If $H$ has at least one edge or has a self-loop, then $\mathcal{F}_{\text {cycle }}$ is VNP-complete under c-reductions. Else it is in $V A C_{0}$.

The next simple fact shows us which cycles are homomorphic to a given graph $H$. Let $n_{0}$ be defined as $n$ if $n$ is even and $n-1$ if $n$ is odd.

Fact 1. Given $H$ a graph with at least one edge, all cycles of length $n_{0}$ are homomorphic to $H$.

It is easy to see that by folding the graph in half we get one path which is trivially homomorphic to an edge. Our hardness proof will only be able to handle cycles of even length. Luckily this is enough to prove hardness.

Lemma 3.1. Let $\mathcal{U H C}_{n_{0}, \text { even }}$ be the graph property of all cycles of length $n_{0}$. Then $\operatorname{GF}\left(K_{n_{0}}, \mathcal{U H C}_{n_{0}, \text { even }}\right)$ is VNP-hard under c-reductions.

Proof. If $n$ is even, we can immediately use the hardness of $\operatorname{GF}\left(K_{n}, \mathcal{U H C}_{n}\right)$ (cf. Theorem 2.1). If $n$ is odd, we use the following reduction. We have given all cycles of length $n-1$ and want to get all cycles of length $n$. We evaluate the polynomial for $K_{n+1}$ and get all cycles of length $n+1$. We can contract one edge with the following argument. We enforce, via taking the homogeneous component of degree one of $x_{n+1,1}$, all cycles to use $x_{n+1,1}$. We then replace $x_{i, n+1}$ by $x_{i, 1}$ for all $i$ and set $x_{n+1,1}$ to one. This gives us all cycles of length $n$ with a factor 2 for every monomial.

To see this let us look at the following argument. Let the edge $(n+1,1)$ be the edge we contract and let $i, j$ be two arbitrary points picked in the graph. If we connect $i, j$ with a path through every point we can complete this into a cycle two different ways. Either with the edge $(1, i),(n+1, j)$ or $(1, j),(n+1, i)$. Notice, that every different choice of $i, j$ will construct a different cycle if we contract 1 and $n+1$.

This concludes our reduction to $\operatorname{GF}\left(K_{n}, \mathcal{U H C}_{n}\right)$. As our circuit can easily divide by two if the polynomial is over an infinite field (see. [21]).

Later proofs will also use the contracting idea from the previous lemma. A simple case distinction will give us the proof of the theorem.

Proof of Theorem 3.1. If $H$ has at least one edge, we know from Fact 1 that all even cycles are homomorphic to $H$ and by this represented in our polynomial. If we take the 
homogeneous components of degree $n_{0}$, we extract all even cycles of length $n_{0}$. This is VNP-hard via the previous Lemma (3.1).

If $H$ has a self-loop, we can map all cycles to the one vertex in $H$. We can then extract the hamiltonian cycles of length $n$ by using the homogeneous degree of $n$ as all cycles are homogeneous to a self-loop.

If $H$ has no edge, our polynomial is the zero polynomial as we cannot map any graph $G$ containing an edge to $H$.

Using Valiant's Criterion, we can prove membership of $\mathcal{F}_{\text {cycle }}$ in VNP (cf.[2]).

\subsection{Cliques}

Here, we will not use cycles in the hardness proof but work directly with the clique polynomial defined by Bürgisser. The complete proof is an easy exercise. In contrast to the other results, we show that computing $\mathcal{F}_{\text {clique }}$ is easy for most choices of $H$.

Theorem 3.2. If $H$ has a self-loop then $\mathcal{F}_{\text {clique }}$ is VNP-complete under c-reductions. Otherwise $\mathcal{F}_{\text {clique }}$ is in $V A C_{0}$.

Proof. Let $H$ have at least one edge and no self-loop. We can use that $H$ has constant size which implies that $H$ has a maximal subgraph which forms a clique or $H$ has no clique. If $H$ has no clique, only a single edge or a single vertex is homomorphic to $H$.

Let us now look at the case for cliques of size $c$. We can compute $\mathcal{F}_{\text {clique }}$ explicit by a brute-force algorithm. The number of monomials can be bound by the following argument. There are $\sum_{i=2}^{c}\left(\begin{array}{c}n \\ i\end{array}\right)$ many different cliques. As we can bound $\left(\begin{array}{l}n \\ i\end{array}\right)$ by $n^{i}$ we get an upper bound of $c n^{c}$ monomials. Further inspection yields, that constant depth, unbounded fan-in circuits of polynomial size are enough to compute all cliques up to size $c$.

Let $H$ now have one self-loop. The fact that all cliques are homomorphic to a given graph with a self-loop tells us that $\mathcal{F}_{\text {clique }}$ contains different monomials for all cliques of size $i$ for $i=1 \ldots, n$. The VNP-hardness follows via Theorem 2.2.

The empty graph has the zero polynomial.

As a polynomial time deterministic machine can easily check if a given instance is a clique, we can use Valiant's Criterion to show membership in VNP.

\subsection{Trees}

As the new characterization of VP had a specific tree structure we want to look at the general problem. In previous sections our polynomial just contained the edges of the graph but for this section we need a slightly different model. If a monomial in our polynomial would select the edges $E^{\prime}$ we also select the vertices $\left\{u, v \mid\{u, v\} \in E^{\prime}\right\}$ in our monomial. In essence, we will also select the vertices forming the edges, giving us polynomials with variables $X=\left\{x_{e} \mid e \in E\right\} \cup\left\{x_{v} \mid v \in V\right\}$. It will be clear later why we need this special form.

Theorem 3.3. If $H$ contains an edge, then $\mathcal{F}_{\text {tree }}$ is VNP-complete under c-reductions. Otherwise $\mathcal{F}_{\text {tree }}$ is in $V A C_{0}$. 


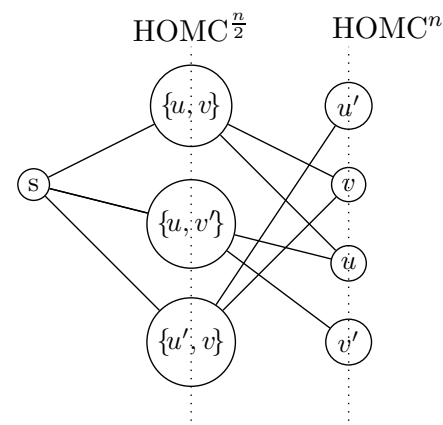

Figure 1: Reduction from Trees to matching

Proof. We use a reduction from connected partial trees to perfect matchings. It is obvious that a tree is always homomorphic to one edge.

We want to compute a matching on a graph given by $(V, E)$. We can build a graph as in Figure 1 from a $K_{n}$ by setting the weight of every edge not given to zero. In detail, our graph has vertices $\{v \in V\} \cup\left\{v_{e} \mid e \in E\right\} \cup\{s\}$. We add the edges $\{(u, v), u\},\{(u, v), v\}$ and $\left\{s, v_{e}\right\}$ for every $e \in E$. Vertices of the form $\left\{v_{e} \mid e \in E\right\}$ will be called edge-vertices in this proof. Now as the vertices are given by our polynomials we can take the homogeneous components over vertices. We take the homogeneous components of degree $n / 2$ over vertices $\left\{v_{e} \mid e \in E\right\}$ and of degree $n$ of vertices $v \in V$. Our matching in the original graph is given by the edges $\left(s, v_{e}\right)$.

Every matching in the original graph has obviously a tree in our graph. Left to prove is the other direction. Given a tree in our graph, we know that only $n / 2$ edge-vertices are selected. As every vertex $v \in V$ has to be connected by an edge, edge-vertices have to go to pairwise different sets of $v \in V$. Hence we can compute a perfect matching which is as hard as computing the permanent.

Valiant's Criterion will again show the membership.

We crucially need the fact that we get the adjacent vertices for free in our homomorphism polynomials. The reader might think restricting the edges out of $s$ might suffice but this is not the case. We could have a path that starts from $s$ goes over an edge-vertex to a vertex $u$ takes the edge from $u$ to some other edge-vertex and continues until we have connected all edge-vertices and all vertices into a path. This is obviously not a matching. If we want to forbid this behaviour, we might want to select all edges outgoing from $s$. This would prevent the above case but the reconstruction of a matching is non trivial.

An interesting fact of the proof is, that it does not use the fact that the graph class only contains trees. Instead we only use that it contains trees. Hence the theorem can be easily extended to other graph classes, provided we look at the homomorphism polynomials which contain edges and the vertices connected to these edges.

Corollary 3.1. Let $\mathcal{C}$ be a graph class containing all trees of size $n$. Then the following theorem holds on homomorphism polynomials containing edge and vertex variables from $\mathcal{C}$ to a given $H$. If $H$ contains an edge, then the homomorphism polynomial is VNPcomplete under c-reductions. Otherwise it is in $V A C_{0}$. 


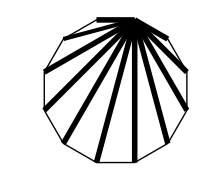

(a) Triangle graph

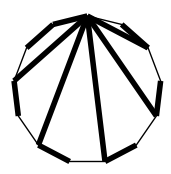

(b) Illustration of graph with buddy vertices

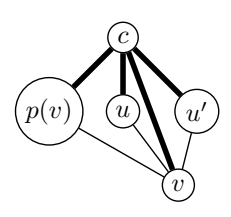

(c)

Figure 2

Proof. We can easily set the weight of every vertex not in our tree to zero and construct the same reduction as in the theorem.

\subsection{Outerplanar Graphs}

Next we will show a dichotomy for outerplanar graphs. We start with the case of a triangle homomorphic to $H$.

Lemma 3.2. If a triangle is homomorphic to $H$ then $\mathcal{F}_{\text {outerplanar }}$ is VNP hard under c-reductions.

Proof. We will reduce to Hamiltonian Cycle by using a construction as in Figure 2a. This means, we pick an arbitrary vertex $c$ and enforce all $n$ outgoing edges from this vertex via homogeneous components. We further enforce the whole graph to have $n+n-3$ edges. The graph given is obviously outerplanar but we still need to proof that no other graph fulfilling our criteria can be outerplanar.

We call the implied order of the graph, the order of the outer circle of vertices starting from the star and ending at it again without any edges crossing. As there are two such orderings let us fix an arbitrary one for every graph. Let us now look at a graph which has not an implied order of the outer vertices. This implies that there exists a vertex $u$ which has degree 4 . With our ordering every vertex (except $c$ up to and including the later defined vertex $v$ has a single parent. Furthermore, let $v$ be the first vertex of degree 4 in this order and let $p(v)$ be the parent of $v$. Notice that by enforcing all $n$ instead of just $n-2$ edges starting at the center, a parent $p(v) \neq c$ has to exist.

Let $u, u^{\prime}$ denote the other vertices adjacent to $v$ different than $p(v)$ and $c$. As we enforced edges from $c$ to every vertex, we can easily see the $K_{2,3}$ with $v, c$ on the one side and $u, u^{\prime}, p(v)$ on the other side. Hence the graph cannot be outerplanar. This implies that every vertex except $c$ and the two neighbouring vertices have degree at most 3. Enforcing the overall number of edges gives us at least degree 3 and hence implies equality.

From this we can reconstruct all cycles in a $K_{n-2}$. We need to remove the center of the star and glue the two vertices on the cycle next to the center together. We do this by a similar argument as in the proof for Lemma 3.1. We evaluate the other enforced edges with one to get all cycles in a $K_{n-2}$ where every monomial is weighted by 2 . Division again gives us the correct polynomial. Taking the homogeneous components as described only increases the circuit by a factor of $n$. 


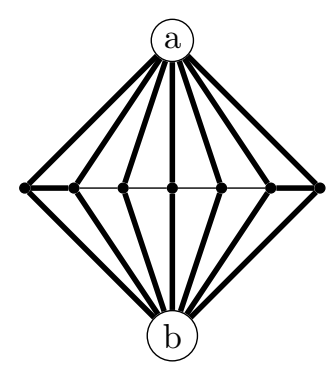

Figure 3: Planar Gadget

Theorem 3.4. If $H$ has an edge then $\mathcal{F}_{\text {outerplanar }}$ is VNP-complete under c-reductions and otherwise trivial.

Proof. To make the graph homomorphic to a single edge we will modify it in the following way. For every vertex $v$, except $c$, we choose a buddy vertex $v^{\prime}$. We enforce the edge between every vertex and his buddy vertex and set the edge between a buddy vertex and $c$ to zero. Additionally, we set all vertices from $v$ to any other non buddy vertex to zero and all edges from a buddy vertex to a different buddy vertex to be zero. In essence this splits every vertex into a left and right part (see Figure $2 \mathrm{~b}$ ). The hardness proof follows from Lemma 3.2 by contracting the edge between a vertex and his buddy vertex. Hence the combined degree of a vertex and his buddy vertex is at most three. Taking the homogeneous components increases the circuit size by a factor of $n$.

We know by [20] that checking if a graph is outerplanar is possible in linear time. With this we can use Valiant's Criterion to show the membership.

\subsection{Planar Graphs}

Lemma 3.3. All graphs isomorphic to Figure 3 with the thick edges fixed and $n+2+$ $2(n+2)$ edges required are all permutations of the vertices $(1, \ldots, n)$.

Proof. Take an embedding in the plane of the graph without any crossings. If we show that every vertex has at most one edge going to the right, it follows that the set of vertices from left to right ordered is a permutation of the vertices.

Let us look at the following subgraph. Let $v$ be a vertex with two right successors $u, u^{\prime}$ and a parent $p(v)$. By construction the parent always exists. We denote the top and bottom vertex by $a$ and $b$ in our graph. We can now build a $K_{3,3}$ minor in the following way. $S_{1}=\{v, a, b\}$ and $S_{2}=\left\{u, u^{\prime}, p^{\prime}\right\}$. As $a$ and $b$ are connected to every vertex we only need to check that $u$ is connected to $u, u^{\prime}$ and $p$ which is by assumption. This proves that via edge deletion our graph would have a $K_{3,3}$ minor if the vertices would not give us a permutation.

Theorem 3.5. If $H$ has an edge then $\mathcal{F}_{\text {planar }}$ is VNP-complete under c-reductions. Otherwise $\mathcal{F}_{\text {planar }}$ is in $V A C_{0}$. 


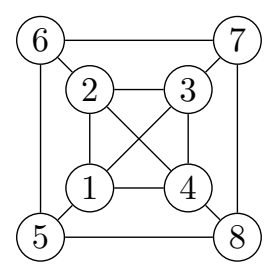

(a) Gadget

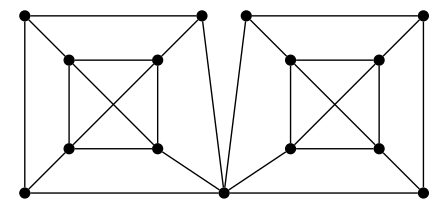

(b) Two Gadgets

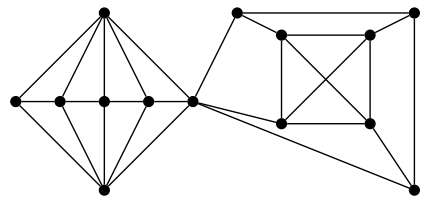

(c) Gadget with planar gadget

Figure 4

Proof. We again glue the second and second to last vertex in our planar gadget together in a similar manner as in the previous constructions to get all cycles from a path. Notice, how these are independent of the order and hence the same for all possible ordering.

However, this graph is not yet homomorphic to a single edge. To accomplish this, we will use a graph of size $2 n$. We, as in the outerplanar case, enforce every vertex, except $a$ and $b$, to have a buddy vertex $u_{v}$. Then we subdivide the edge $(a, v)$ and $(b, v)$ for every original, meaning none buddy, vertex $v$ with a new vertex $v_{a}^{\prime}, v_{b}^{\prime}$ respectively. This will give us for every part a square consisting of the vertices $a, v, v_{a}^{\prime}, u_{v}$ and the square $b, v, v_{b}^{\prime}, u_{v}$.

Now it is easy to see that we can fold $a$ to $b$ which leaves us with a grid of height one. A grid can be easily folded to one edge. The size of the circuit is increased by a factor of at most $2 n$.

As testing planarity is easy, we can use Valiant's Criterion to show membership.

\subsection{Genus $k$ graphs}

Graph embeddings are one of the major relaxations of planarity. For this we find a surface of a specific type such that a graph can be embedded in this surface without any crossing edges. If we want to increase the orientable genus of a surface by one, we can glue a handle onto it which edges can use without crossing other edges. We call a graph a genus $k$ graph if there exists a surface of orientable genus $k$ such that $G$ can be embedded in this surface and $k$ is minimal. Notice, that a genus 0 graph is planar. While the topic of graph genus is vast, we will mostly use theorems as a blackbox and only reason about graphs of genus zero and one. For a detailed coverage of the topic, the reader is referred to 8$]$.

With the planar result in place we can use the simple proof strategy. Construct a genus $k$ graph where we append the planar construction. In this way the genus bound will ensure that our planar gadget gives us all permutation of vertices as long as the connection of these two graphs will not reduce the genus.

Lemma 3.4. The graph in Figure 40 has genus one.

Proof. We can use the given embedding with one handle for the crossing in the middle to show an upper bound of one. 
We again construct a $K_{3,3}$ with the sets $S_{1}=\left\{2,1,6^{\prime}\right\}, S_{2}=\left\{3,4,7^{\prime}\right\}$ where $6^{\prime}$ is the vertex constructed from contracting the edge $(5,6)$ and $7^{\prime}$ from the edge $(7,8)$. And hence the graph is not planar and has a lower bound for the genus of one.

The next theorem shows how we can glue graphs together to increase the genus in a predictable way.

Definition 3.1 ([19]). $G$ is a vertex amalgam of $H_{1}, H_{2}$ if $G$ is obtained from disjoint graphs $H_{1}$ and $H_{2}$ where we identify one vertex form $H_{1}$ with one vertex from $\mathrm{H}_{2}$.

With this we restate a theorem from Miller [19] to compute the genus of a given graph.

Theorem 3.6 ([19]). Let $\gamma(G)$ be the orientable genus of a graph $G$. Let $G$ be constructed from vertex amalgams of graphs $G_{1}, \ldots, G_{n}$. Then $\gamma(G)=\sum_{i=1}^{n} \gamma\left(G_{i}\right)$.

This now gives us immediately the result that a graph constructed as in Figure $4 \mathrm{~b}$ with $k$ gadgets has genus $k$.

Theorem 3.7. If $H$ has an edge then $\mathcal{F}_{\text {genus }(k)}$ is VNP-complete under c-reductions for any $k$. Otherwise $\mathcal{F}_{\text {genus }(k)}$ is in $V A C_{0}$.

Proof. With Theorem 3.6, Lemma 3.3 and the construction in Figure 4 we are almost done. Because we enforced a genus $k$ graph to occur all graphs that homomorphic to the planar gadget have genus zero and hence be planar.

The only thing left to do is to modify our graphs such that they are homomorphic to an edge without violating the properties. It is clear that we can fold our genus one gadgets together. If we then subdivide the edge $(1,3)$ and $(2,4)$ (which keeps our block property) we can first fold 7 to 5 and 3 to 1 . Folding then again 6 to 8 and 2 to 4 we get a square with two dangling edges. The dangling edges can be folded onto the square and the square is homomorphic to one edge. This construction increases the size of the circuit at most by a factor of $14 k+2 n$. As testing for a fixed genus is in NP, we can use Valiant's Criterion to show membership.

\section{Conclusion}

We have shown many dichotomy results for different graph classes but some classes are still open. We want to especially mention the case of our graph class being the class of trees. It is known that we can use Kirchoff's Theorem to find all spanning trees of a given graph. This, however, does not include monomials of total degree less than $n-1$ which our polynomials include. From the algebraic view, the knowledge ends here. In the counting view, where we solve the task of counting all trees in a graph, a bit more is known. Goldberg and Jerrum [13] showed that counting the number of subtrees that are distinct up to isomorphism is \#P-complete. This, combined with our dichotomy for trees including the vertices, gives us a strong indication that the similar problem is VNP-hard in the algebraic world. 
A different expansion of these results would be the case of bounded treewidth. As mentioned earlier, in the counting version the case of bounded treewidth is indeed the most general form and completely characterizes the easy and hard instances of counting graph homomorphisms. Additionally, recent advancements showed that graph homomorphisms of a specific type characterize VP. Can homomorphism from graph classes parameterized by treewidth, similar to the counting case, be used for a complete characterization of VP and VNP?

An interesting research direction would be the case of disconnected graph properties. Rugy-Altherre looked at the property that any graph is homomorphic to a given graph $H$. This includes disconnected graphs with connected components larger than one vertex. We instead only looked at restricted homomorphisms where one major connected component exists. It is unclear to the author if our proofs could be adapted to this case.

Acknowledgments I want to thank my doctoral advisor M. Bläser for his guidance. I additionally want to thank R. Curticapean for many discussions on the counting versions on problems and B. V. Raghavendra Rao for introducing me to this topic.

\section{References}

[1] A. A. Bulatov and M. Grohe. The complexity of partition functions. Theor. Comput. Sci., 348(2-3):148-186, 2005.

[2] P. Bürgisser. Completeness and reduction in algebraic complexity theory, volume 7. Springer, 2000.

[3] J. Cai, X. Chen, and P. Lu. Graph homomorphisms with complex values: A dichotomy theorem. SIAM J. Comput., 42(3):924-1029, 2013.

[4] C. Chekuri and A. Rajaraman. Conjunctive query containment revisited. Theor. Comput. Sci., 239(2):211-229, 2000.

[5] V. Dalmau and P. Jonsson. The complexity of counting homomorphisms seen from the other side. Theor. Comput. Sci., 329(1-3):315-323, 2004.

[6] V. Dalmau, P. G. Kolaitis, and M. Y. Vardi. Constraint satisfaction, bounded treewidth, and finite-variable logics. In $C P$, pages 310-326, 2002.

[7] N. de Rugy-Altherre. A dichotomy theorem for homomorphism polynomials. In MFCS, volume 7464 of LNCS, pages 308-322. Springer, 2012.

[8] R. Diestel. Graph Theory. Springer-Verlag Berlin and Heidelberg GmbH \& Company KG, 2000.

[9] A. Durand, M. Mahajan, G. Malod, N. de Rugy-Althere, and N. Saurabh. Homomorphism polynomials complete for VP. FSTTCS, 2014. to appear. 
[10] M. E. Dyer and C. S. Greenhill. The complexity of counting graph homomorphisms (extended abstract). In SODA, pages 246-255, 2000.

[11] E. C. Freuder. Complexity of k-tree structured constraint satisfaction problems. In $A A A I$, pages $4-9,1990$.

[12] L. A. Goldberg, M. Grohe, M. Jerrum, and M. Thurley. A complexity dichotomy for partition functions with mixed signs. SIAM J. Comput., 39(7):3336-3402, 2010.

[13] L. A. Goldberg and M. Jerrum. Counting unlabelled subtrees of a tree is \#pcomplete. LMS J. Comput. Math, 3:117-124, 2000.

[14] M. Grohe. The complexity of homomorphism and constraint satisfaction problems seen from the other side. J. ACM, 54(1), 2007.

[15] M. Grohe and M. Thurley. Counting homomorphisms and partition functions. Model Theoretic Methods in Finite Combinatorics, 558:243-292, 2011.

[16] P. Hell and J. Nešetrril. On the complexity of h-coloring. Journal of Combinatorial Theory, Series B, 48(1):92 - 110, 1990.

[17] P. Hell and J. Nešetřil. Graphs and homomorphisms, volume 28. Oxford University Press Oxford, 2004.

[18] M. Mahajan and B. V. R. Rao. Small space analogues of valiant's classes and the limitations of skew formulas. Computational Complexity, 22(1):1-38, 2013.

[19] G. L. Miller. An additivity theorem for the genus of a graph. J. Comb. Theory, Ser. $B, 43(1): 25-47,1987$.

[20] S. L. Mitchell. Linear algorithms to recognize outerplanar and maximal outerplanar graphs. Information Processing Letters, 9(5):229-232, 1979.

[21] V. Strassen. Vermeidung von divisionen. Journal für die reine und angewandte Mathematik, 264:184-202, 1973.

[22] L. G. Valiant. Completeness classes in algebra. STOC '79, pages 249-261, 1979. 